\title{
Direct Interbranch Relaxation of Polaritons in a Microcavity with Embedded CdSe/(Cd,Mg)Se Quantum Wells
}

\author{
KRZYSZTOF SAWICKI ${ }^{10},{ }^{1}$ MAGDALENA JURCZAK, ${ }^{1}$ \\ WOJCIECH PACUSKI (i), ${ }^{1}$ and JAN SUFFCZYŃSKI (i] ${ }^{1,2}$ \\ 1.-Faculty of Physics, Institute of Experimental Physics, University of Warsaw, Pasteura 5 St., \\ 02-093 Warsaw, Poland. 2.—e-mail: Jan.Suffczynski@fuw.edu.pl
}

\begin{abstract}
In polariton microcavities, the upper polariton branch is often hardly observable in reflectivity or photoluminescence measurements, which hampers reliable determination of the vacuum Rabi splitting value. We studied the photoluminescence excitation of a semiconductor microcavity with eight embedded $\mathrm{CdSe} /(\mathrm{Cd}, \mathrm{Mg})$ Se quantum wells and observed a maximum in the emission from the lower polariton branch when the energy of the excitation was tuned to that of the upper polariton branch. This indicates that, in microcavities, where the upper polariton branch is hardly observable but interbranch polariton relaxation is efficient, the Rabi splitting value can be determined based on the energy difference between the absorption and emission maxima in photoluminescence excitation measurements. The Rabi splitting value of the studied sample as determined by reflectivity measurements was $18 \pm 2 \mathrm{meV}$. Strong coupling conditions allow us to observe lasing with a relatively low threshold of $0.24 \mathrm{~kW} \mathrm{~cm}^{-2}$.
\end{abstract}

Key words: Optical microcavity, exciton-polariton,

photoluminescence excitation, CdSe, molecular beam epitaxy, II-VI semiconductor

\section{INTRODUCTION}

Strong light-matter coupling gives rise to the formation of a mixed quasiparticle called the exciton-polariton as the superposition of an exciton and photon. ${ }^{1}$ This effect is particularly distinct in microcavity structures, where the optical mode interacts with excitons confined in quantum wells (QWs). ${ }^{2}$ Typically, strong coupling conditions are manifested by anticrossing behavior, which can be studied, e.g., using reflectivity or photoluminescence measurements. Anticrossing is a consequence of coherent energy exchange between the QW exciton and the cavity mode, which leads to the formation of two new eigenstates: the upper polariton branch (UPB) and the lower polariton branch (LPB). The energy

(Received January 18, 2020; accepted May 14, 2020;

published online June 2, 2020) difference between the UPB and LPB at resonance between the mode and the exciton determines the exciton-photon coupling parameter, which is called the vacuum Rabi splitting. If the frequency corresponding to the Rabi splitting is larger than the exciton and cavity photon decay rates, the system enters a strong coupling regime. ${ }^{2}$ However, when the damping rates dominate, the system operates in weak coupling conditions, where polariton effects are negligible.

Experimentally, the magnitude of the Rabi splitting is typically determined by analysis of the energy of the UPB and LPB in spectra recorded as a function of the detuning between the QW exciton and the microcavity mode. ${ }^{2}$ However, the UPB is often barely visible in such reflectivity measurements, and due to a quick polariton relaxation, it is rarely observable at all in photoluminescence measurements. This was the case for high-quality II-VI microcavities studied previously, ${ }^{3-6}$ and in 
particular the ZnTe-based microcavity with embedded $\mathrm{CdSe} /(\mathrm{Cd}, \mathrm{Mg}) \mathrm{Se} \mathrm{QWs}$ studied in our recent work. ${ }^{7}$

In the work presented herein, we determined the energy of the UPB as well as the energy of the vacuum Rabi splitting in a CdSe-based microcavity structure by photoluminescence excitation (PLE) measurements. The results show that the emission from the LPB is enhanced when the excitation energy is tuned to the energy of the UPB. This points toward efficient direct interbranch polariton relaxation, i.e., the process of polariton transfer from the upper to lower polariton branch with $k=0$. We expect that this process should be most efficient in the vicinity of zero QW-mode detuning. ${ }^{8}$ Precise determination of the UPB energy for near-zero QWmode detuning allows us to establish the value of the Rabi splitting in a reliable way. The presented method could be particularly useful for the determination of the coupling parameters for microcavities with embedded layers of transition-metal dichalcogenides ${ }^{9,10}$ or organic materials, ${ }^{11,12}$ for which the Rabi splitting value is usually relatively large.

\section{SAMPLE DESIGN}

The system under study is made of II-VI materials, which exhibit a smaller exciton Bohr radius ${ }^{13}$ and larger exciton oscillator strength than their III$\mathrm{V}$ counterparts. To reduce the density of dislocations arising during molecular beam epitaxy (MBE) growth, we used three consecutively grown buffer layers: GaAs (200 nm thickness), ZnSe (70 nm), and ZnTe $(1000 \mathrm{~nm})$. The microcavity consisted of two distributed Bragg reflectors (DBRs) made of 16 and 13.5 pairs of alternating refractive index layers, forming the bottom and top mirror, respectively. The 130-nm-thick (Cd,Mg)Se microcavity, placed between the mirrors, contained eight 20 -nm-wide CdSe quantum wells separated by 1 -nm-wide MgSe barriers. ${ }^{14,15}$ The QWs were placed at the antinode of the electric field of the $\lambda / 2$ microcavity. A relatively high number of QWs was designed to enhance the strength of the photon-exciton coupling ${ }^{16,17}$ and boost the optical performance of the structure (i.e., to enable strong coupling conditions up to elevated temperatures and lower the threshold for the onset of polariton lasing). A schematic of the structure is illustrated in Fig. 1a.

The bottom and top DBRs were made of alternating layers of $\mathrm{ZnTe}$ (with a high refractive index of 2.95) and a short-period ZnTe I MgTe I MgSe I MgTe superlattice (with a low refractive index of 2.63). This superlattice, matched to $\mathrm{ZnTe}$, was designed using transfer-matrix method calculations (shown in Supplementary Note 1) and is similar to the ZnTelMgSelZnTelMgTe structure reported in Refs. ${ }^{6,18,19}$, although in our case a possible reaction of $\mathrm{Zn}$ and $\mathrm{Se}$ atoms is avoided thanks to the improved layer sequence (with the MgSe layer being surrounded by MgTe instead of ZnTe layers). The nominal thickness of the DBR layers $\left(d_{\text {high }}=61\right.$ $\mathrm{nm}$ and $d_{\text {low }}=75 \mathrm{~nm}$ ) was adjusted to achieve a stopband centered at $\lambda_{0}=730 \mathrm{~nm}$. The layer thickness was monitored by in situ reflectivity during sample growth, resulting in a proper thickness of the layers as confirmed by scanning electron microscopy (SEM) of the sample cross-section (Fig. 1b). The sharp interfaces indicate a good quality of the structure.

To create a wedge-type cavity and thereby enable tuning of the energy of the cavity mode by selection of the position on the sample surface, the sample was not rotated during growth. The presented structure allows for a broad range of spectral tuning (between $600 \mathrm{~nm}$ and $750 \mathrm{~nm}$ ) of the mode thanks to the 3-inch-diameter GaAs:Si substrate (Fig. 1c). Reflectivity measurements at room temperature reveal that the stopband is wide and symmetrical (see Supplementary Note 1) for a whole range of mode-exciton detuning. The linewidth of the mode determined by microphotoluminescence ( $\mu$-PL) is about $3 \mathrm{meV}$. The microcavity quality factor exceeds 600 .

\section{EXPERIMENT}

All experiments were performed in a liquid-helium flow cryostat, allowing us to obtain temperatures of about $8 \mathrm{~K}$. As an excitation source, we used either a pulsed Ti:sapphire laser coupled to an optical parametric oscillator $\left(\lambda_{\mathrm{exc}}=600 \mathrm{~nm}, P_{\mathrm{exc}}\right.$ of up to $40 \mu \mathrm{W}$ ) or a continuous-wave (cw) Ti:sapphire laser tuned in the range between $720 \mathrm{~nm}$ and $800 \mathrm{~nm}\left(P_{\text {exc }}=1.3 \mathrm{~mW}\right)$. A Peltier-cooled chargecoupled device (CCD) camera, coupled to a spectrometer, served as the detector. A halogen lamp was employed as the light source in the reflectivity measurements. A microscope objective allowed us to address and collect the signal from a spot on the sample surface with a diameter of about $1.5 \mu \mathrm{m}$.

\section{RESULTS}

We start with a characterization of the sample by reflectivity measurements at $8 \mathrm{~K}$. A series of reflectivity spectra at normal incidence, taken from consecutive positions on the sample surface along the microcavity thickness gradient, are shown in Fig. 2. The presented map reveals anticrossing between exciton and cavity mode, providing clear evidence of strong light-matter coupling conditions in the studied structure. We trace the minima in the reflectivity spectra, obtaining energies of the LPB and UPB as a function of detuning. Next, we simulate the LPB and UPB energies using a twocoupled-oscillator model, ${ }^{2}$ which allows us to determine a Rabi splitting value of $18 \pm 2 \mathrm{meV}$. Due to the possible coupling of the QWs, the exciton oscillator strength and, consequently, the value of the Rabi splitting, might be slightly lower than in a structure with uncoupled QWs of the same 


\section{(a)}

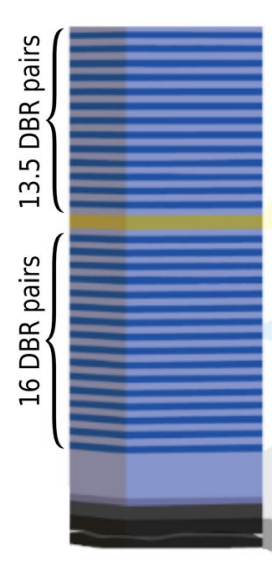

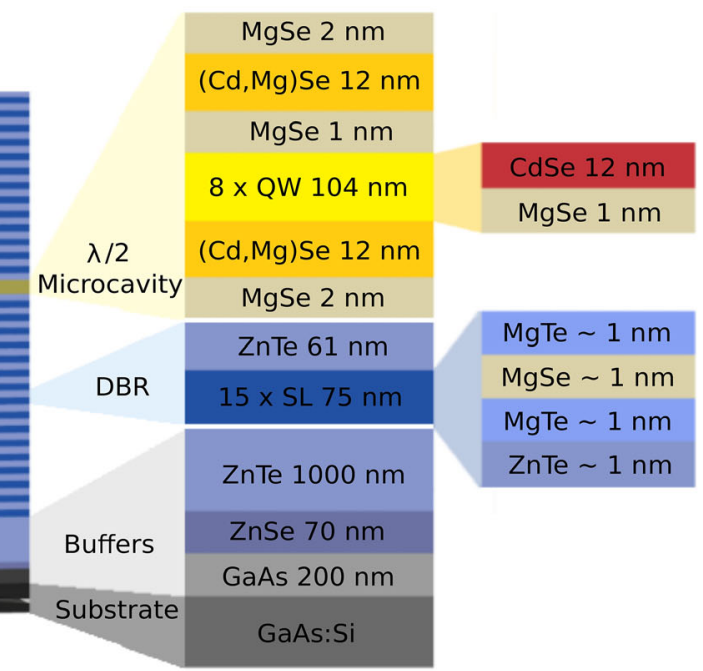

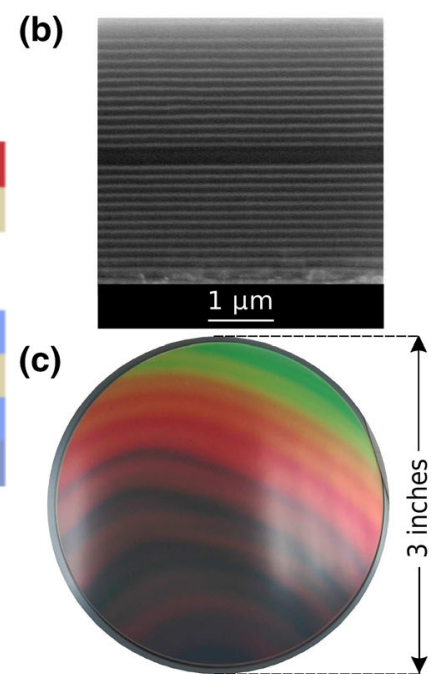

Fig. 1. Structure design: (a) Sketch of sample comprising $\lambda / 2(\mathrm{Cd}, \mathrm{Mg})$ Se microcavity with eight embedded CdSe/(Cd,Mg)Se quantum wells (QWs), surrounded by lattice-matched, ZnTe-based distributed Bragg reflectors (DBRs). Nominal values of layer thicknesses are indicated. (b) SEM image of sample cross-section in the region of the microcavity. (c) Photograph of the 3-inch wafer. The multicolor pattern proves a wide range of mode spectral tuning (Color figure online).

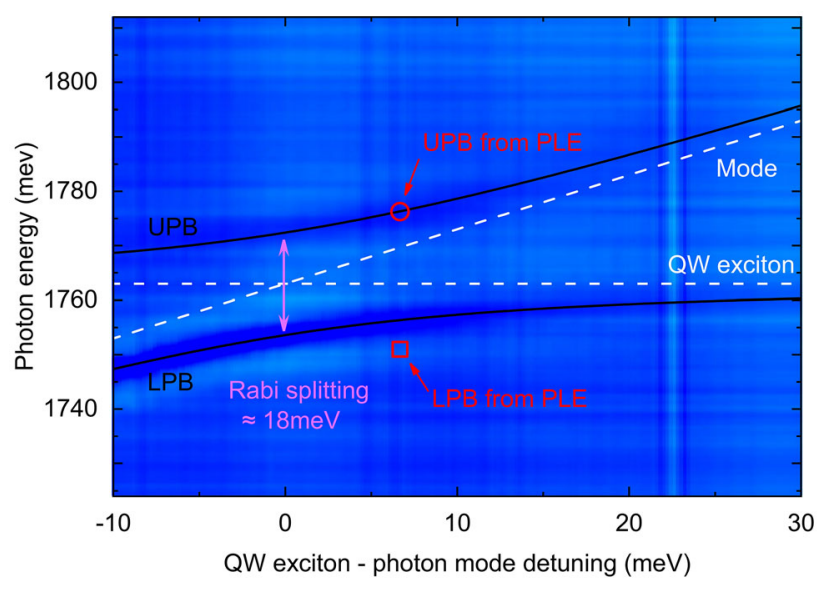

Fig. 2. Reflectivity map at $k_{\|}=0$ at $8 \mathrm{~K}$ as a function of $\mathrm{QW}$ excitonmode detuning. White dashed lines indicate the energies of the uncoupled QW exciton and cavity mode. Black lines represent the position of the calculated upper and lower polariton branches. Modeling of energies of optical transitions observed in the experiment provides a vacuum Rabi splitting value of $18 \pm 2 \mathrm{meV}$. Vertical and horizontal shadow-like lines are artifacts related to the measurement setup.

thickness; however, the present value is still relatively large.

Next, we recorded the $\mu$-PL spectra for consecutive excitation energies (Fig. 3) at a position on the sample where the mode-exciton detuning was close to zero. We observed that the emission from the LPB (at $1750 \mathrm{meV}$ ) was enhanced when the excitation energy was around $1776 \mathrm{meV}$ and when it approached the energy of the LPB (around $1760 \mathrm{meV})$. We attribute the maximum at $1776 \mathrm{meV}$ to the increased absorption of the excitation due to the resonance with the UPB. Both the UPB and LPB occur in the stopband region, as evidenced by two minima in the reflectivity

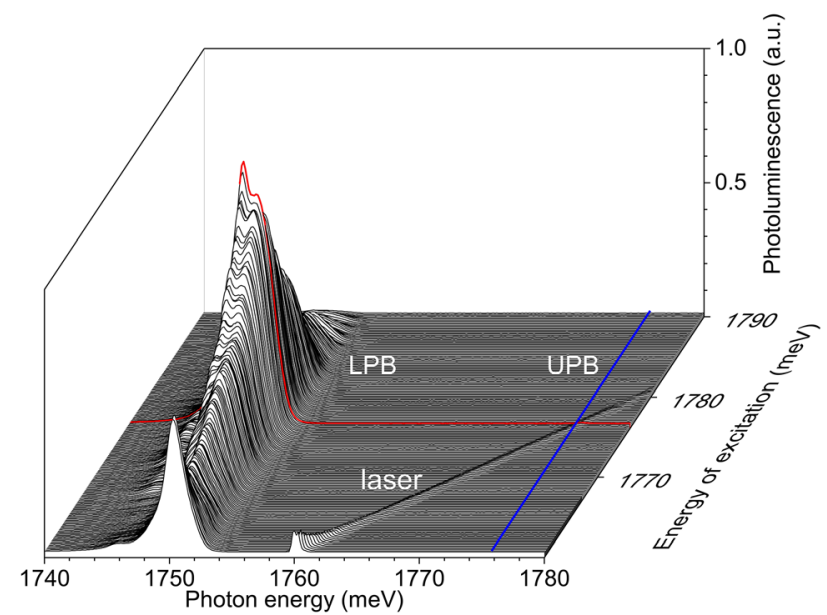

Fig. 3. Photoluminescence spectra versus excitation energy at $8 \mathrm{~K}$. Red curve highlights the spectrum for which the maximum intensity of the lower polariton branch (LPB) is obtained. The blue line indicates the energy of the upper polariton branch (UPB). The maximum emission from the LPB is observed when the energy of the excitation is tuned to the energy of the UPB (Color figure online).

spectrum (Fig. 4b). The locally increased absorption associated with the UPB allows us to stimulate emission from the LPB through the UPB. When the excitation energy is tuned to the UPB, we observe a maximum of the emission from the bottom of the LPB due to the efficient direct interbranch polariton relaxation.

Figure 4 compares the reflectivity, PLE, and $\mu$-PL spectra registered in the same region of the sample. The maximum centered at around $1776 \mathrm{meV}$ can be clearly seen in Fig. 4a. Two spectrally wide dips, evidenced in the reflectivity spectrum (Fig. 4b) at around $1752 \mathrm{meV}$ and $1776 \mathrm{meV}$, mark the position of the LPB and UPB, respectively. The PL spectrum 

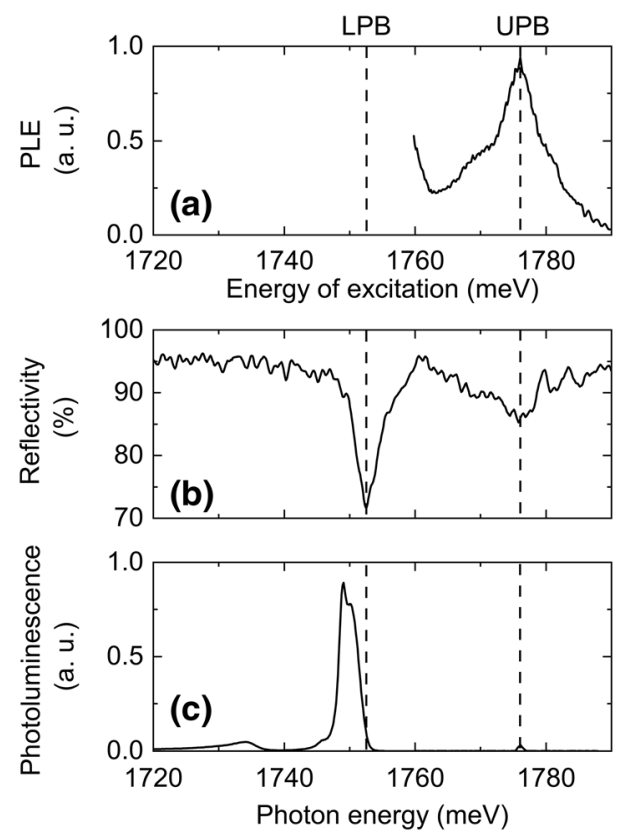

Fig. 4. Optical measurements at 8 K. A comparison of (a) PLE, (b) reflectivity, and (c) $\mu$-PL spectra taken at the point of the sample where the QW exciton and cavity mode approach resonance.

in Fig. 4c reaches a maximum at around $1750 \mathrm{meV}$, representing the emission from the LPB.

The spectra presented in Fig. 4 indicate the agreement of the UPB energy obtained from the reflectivity and PLE measurements. We attribute a redshift of the LPB energy in the $\mu$-PL spectrum (Fig. 4c) with respect to the LPB dip in the reflectivity spectrum (Fig. 4b) to the Stokes shift, which is the energy difference between the maximum absorption and the PL transitions. ${ }^{20}$ Reflectivity probes the maximum of the optically active density of states, while PL occurs from the states in the lowenergy tail of the absorption band. The relatively large value of the shift results from the relatively broad absorption line of the LPB. Figure 4 also enables us to link the increased emission of the LPB observed when the excitation energy approaches $1760 \mathrm{meV}$ to direct excitation of the LPB through LPB states, extended towards higher energy by disorder-driven broadening.

These results demonstrate that PLE measurements can provide an accurate method for determination of the UPB energy. This method should be particularly useful in cases where a high quality factor of the microcavity precludes direct observation of the UPB by photoluminescence or reflectivity measurements, and the Rabi splitting value is large enough to ensure efficient interbranch polariton relaxation with the emission of the LO phonon (25.9 meV in case of CdSe). Previously, interbranch polariton relaxation assisted by emission of $\mathrm{LO}$ phonons was reported in optically or electrically pumped III-V polariton microcavities. ${ }^{21-23}$

Determination of the Rabi splitting value using solely the PLE method would require systematic

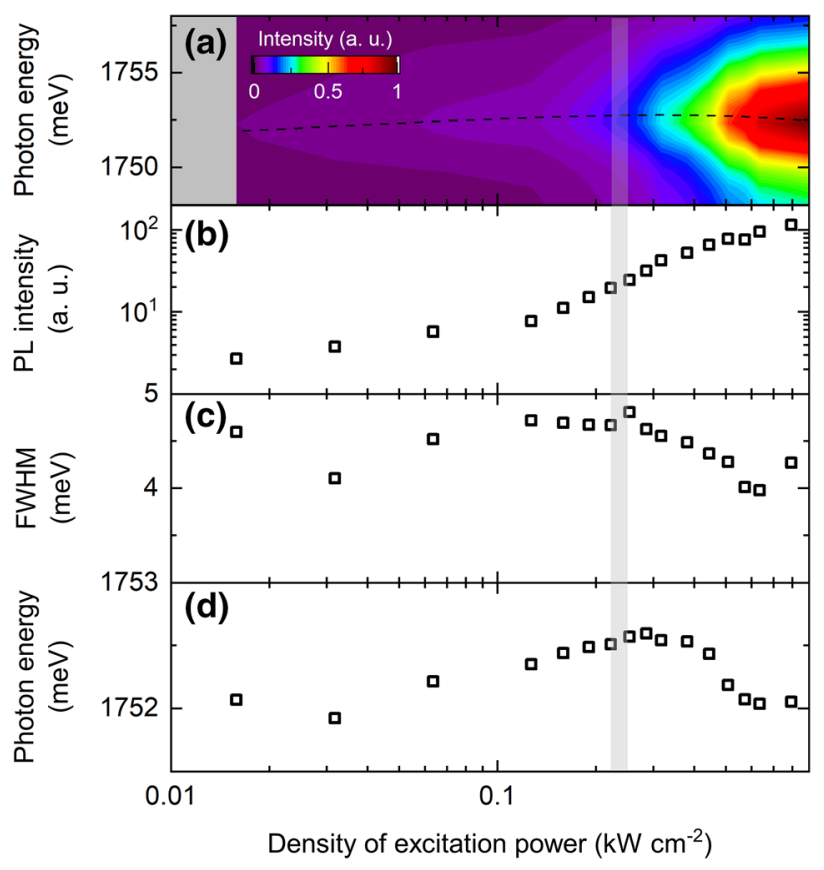

Fig. 5. Excitation power-dependent emission properties at $8 \mathrm{~K}$ : (a) contour map, (b) integrated intensity, (c) linewidth, and (d) energy of LPB emission versus excitation power density. The lasing threshold is $0.24 \mathrm{~kW} \mathrm{~cm}^{-2}$.

measurements to trace the energies of the UPB and LPB as functions of the QW exciton-mode detuning, thus at consecutive points on the sample along the gradient of microcavity thickness. This gradient direction might be established, for instance, by reflectivity mapping at room-temperature conditions, where polariton effects are negligible. Since we do not expect any other emission lines apart from the upper and lower polariton branches in the system under consideration to exhibit nonlinear energy dependence on detuning, LPB and the UBP transitions and their energy dependence on excitonmode detuning can be reliably determined in this way. This will be the subject of a further study. Strong coupling conditions, as confirmed by the anticrossing behavior of the lines in the reflectivity experiment (Fig. 2), allowed us to achieve lasing with a relatively low threshold in the emission from the studied structure. Figure 5 shows a contour map, integrated intensity, linewidth, and spectral position of the LPB emission as a function of the excitation power density under nonresonant, pulsed excitation. The respective emission spectra are shown in Supplementary Note 2. The nonlinear threshold dependence in the emission intensity, associated with a narrowing and blueshift of the spectral line observed under strong coupling conditions, indicate the presence of polariton lasing. ${ }^{24,25}$ The lower lasing threshold (about $0.24 \mathrm{~kW} \mathrm{~cm}^{-2}$, compared with $0.75 \mathrm{~kW} \mathrm{~cm}^{-2}$ ) and larger blueshift than in our previous work on CdSe QWs in a microcavity (Ref. 7) result from the larger number of 
QWs herein and the resulting higher light-matter coupling constant. ${ }^{16}$ In the case of II-VI-based systems, the polariton lasing properties are typically affected by the relatively high degree of crystalline disorder in the structure. This is also the case for the sample examined in this work, where disorder in the photonic potential, introduced spontaneously on the sample growth level, provides additional light confinement in the microcavity plane. As a consequence, the reservoir of photogenerated excitons couples to discrete photonic modes, instead of a continuum of states, as would be the case in an ideal planar microcavity.

This limits fluctuations of the polariton emission energy and possible effects on the linewidth and blueshift of the emission line. Only a weak narrowing of the emission line at the polariton laser threshold was reported in our previous work devoted to lasing from photonic traps in a CdSebased microcavity, ${ }^{7}$ and also in III-V micropillars. ${ }^{26}$

The density of polaritons in a pulsed excitation regime is much greater than in the case of $\mathrm{cw}$ pumping $\left(10^{11}\right.$ excitons $/ \mathrm{cm}^{2}$ per excitation pulse lasting effectively $20 \mathrm{ps}^{27}$ versus around $10^{9}$ excitons $/ \mathrm{cm}^{2}$ per a corresponding period of $20 \mathrm{ps}$ under cw pumping; photon to electron-hole pair conversion efficiency of 0.001 assumed $^{7}$ ). This leads to a slightly higher LPB energy in the case of pulsed pumping, due to polariton-polariton and polaritonexciton reservoir interactions.

\section{CONCLUSIONS}

We present a study of the optical properties of a ZnTe-based microcavity structure with eight embedded $\mathrm{CdSe} /(\mathrm{Cd}, \mathrm{Mg}) \mathrm{Se} \mathrm{QWs}$. The sample used for the investigation shows a relatively large vacuum Rabi splitting value of $18 \mathrm{meV}$. The upper polariton branch is seen in the reflectivity and photoluminescence excitation spectra, but cannot be found directly in photoluminescence measurements. This work demonstrates that photoluminescence excitation provides an alternative method for confirming strong coupling conditions and estimating the vacuum Rabi splitting value in cases when the UPB cannot be observed using typical optical, e.g., photoluminescence or reflectivity, measurements. The presented method for the determination of the vacuum Rabi splitting value by photoluminescence excitation can be applied for polariton systems with large Rabi splitting values, such as microcavities with $\mathrm{ZnO}$, GaN, dichalcogenides, or organic layers serving as an active material. The power-dependent photoluminescence measurements at low temperatures reveal polariton lasing with a relatively low threshold of $0.24 \mathrm{~kW} \mathrm{~cm}^{-2}$.

\section{ACKNOWLEDGMENTS}

The authors thank T. Kazimierczuk for technical support and are grateful to J. Beaumariage and D. Snoke for inspiring discussions. This work was partially supported by the Polish National Science Center under decisions DEC-2013/10/E/ST3/00215, DEC-2015/18/E/ST3/00559, DEC-2017/25/N/ST3/ 00465 . The research was carried out with the use of CePT, CeZaMat, and NLTK infrastructures financed by the European Union-the European Regional Development Fund within the Operational Programme "Innovative economy" for 2007-2013.

\section{OPEN ACCESS}

This article is licensed under a Creative Commons Attribution 4.0 International License, which permits use, sharing, adaptation, distribution and reproduction in any medium or format, as long as you give appropriate credit to the original author(s) and the source, provide a link to the Creative Commons licence, and indicate if changes were made. The images or other third party material in this article are included in the article's Creative Commons licence, unless indicated otherwise in a credit line to the material. If material is not included in the article's Creative Commons licence and your intended use is not permitted by statutory regulation or exceeds the permitted use, you will need to obtain permission directly from the copyright holder. To view a copy of this licence, visit http://creativecom mons.org/licenses/by/4.0/.

\section{ELECTRONIC SUPPLEMENTARY MATERIAL}

The online version of this article (https://doi.org/ 10.1007/s11664-020-08223-6) contains supplementary material, which is available to authorized users.

\section{REFERENCES}

1. J.J. Hopfield, Phys. Rev. 112, 1555 (1958).

2. C. Weisbuch, M. Nishioka, A. Ishikawa, and Y. Arakawa, Phys. Rev. Lett. 69, 3314 (1992).

3. L.S. Dang, D. Heger, R. André, F. Bœuf, and R. Romestain, Phys. Rev. Lett. 81, 3920 (1998).

4. H. Deng, G. Weihs, C. Santori, J. Bloch, and Y. Yamamoto, Science 298, 199 (2002).

5. T. Klein, S. Klembt, E. Durupt, C. Kruse, D. Hommel, and M. Richard, Appl. Phys. Lett. 107, 071101 (2015).

6. J.-G. Rousset, B. Pietka, M. Król, R. Mirek, K. Lekenta, J. Szczytko, J. Borysiuk, J. Suffczyński, T. Kazimierczuk, M. Goryca, T. Smoleński, P. Kossacki, M. Nawrocki, and W. Pacuski, Appl. Phys. Lett. 107, 201109 (2015).

7. K. Sawicki, J.-G. Rousset, R. Rudniewski, W. Pacuski, M. Ściesiek, T. Kazimierczuk, K. Sobczak, J. Borysiuk, M. Nawrocki, and J. Suffczyński, Commun. Phys. 2, 38 (2019).

8. M. Martin, D. Ballarini, A. Amo, L. Viña, and R. André, Superlattice Microsyst. 41, 328 (2007).

9. S. Gambino, M. Mazzeo, A. Genco, O. Di Stefano, S. Savasta, S. Patané, D. Ballarini, F. Mangione, G. Lerario, D. Sanvitto, and G. Gigli, ACS Photon. 1, 1042 (2014).

10. X. Liu, T. Galfsky, Z. Sun, F. Xia, E. Lin, Y.-H. Lee, S. KénaCohen, and V.M. Menon, Nat. Photon. 9, 30 (2015). 
11. S. Kéna-Cohen, M. Davanço, and S.R. Forrest, Phys. Rev. Lett. 101, 116401 (2008).

12. K.S. Daskalakis, S.A. Maier, R. Murray, and S. Kéna-Cohen, Nat. Mater. 13, 271 (2014).

13. P. Mushonga, M.O. Onani, A.M. Madiehe, and M. Meyer, J. Nanomater. 414, 869284 (2012)

14. R. Rudniewski, J.-G. Rousset, E. Janik, P. Kossacki, A. Golnik, M. Nawrocki, and W. Pacuski, Acta Phys. Pol. A 126, 1167 (2014).

15. J.-G. Rousset, J. Kobak, E. Janik, T. Jakubczyk, R. Rudniewski, P. Piotrowski, M. Ściesiek, J. Borysiuk, T. Slupiński, A. Golnik, P. Kossacki, M. Nawrocki, and W. Pacuski, J. Cryst. Growth 401, 499 (2014).

16. V. Savona, L. Andreani, P. Schwendimann, and A. Quattropani, Solid State Commun. 93, 733 (1995).

17. M. Saba, C. Ciuti, J. Bloch, V. Thierry-Mieg, R. Andre, L.S. Dang, S. Kundermann, A. Mura, G. Bongiovanni, J.L. Staehli, and B. Deveaud, Nature 414, 731 (2001).

18. W. Pacuski, C. Kruse, S. Figge, and D. Hommel, Appl. Phys. Lett. 94, 191108 (2009).

19. M. Ściesiek, W. Pacuski, J.-G. Rousset, M. Parlińska-Wojtan, A. Golnik, and J. Suffczyński, Cryst. Growth Des. 17, 3716 (2017).

20. R. Spiegel, G. Bacher, A. Forchel, B. Jobst, D. Hommel, and G. Landwehr, Phys. Rev. B 55, 9866 (1997).
21. A. Delteil, A. Vasanelli, P. Jouy, D. Barate, J.C. Moreno, R. Teissier, A.N. Baranov, and C. Sirtori, Phys. Rev. B 83, 081404 (2011).

22. R. Colombelli and J.-M. Manceau, Phys. Rev. X 5, 011031 (2015).

23. J.-M. Manceau, N.-L. Tran, G. Biasiol, T. Laurent, I. Sagnes, G. Beaudoin, S. De Liberato, I. Carusotto, and R. Colombelli, Appl. Phys. Lett. 112, 191106 (2018).

24. J. Kasprzak, M. Richard, S. Kundermann, A. Baas, P. Jeambrun, J.M.J. Keeling, F.M. Marchetti, M.H. Szymańska, R. André, J.L. Staehli, V. Savona, P.B. Littlewood, B. Deveaud, and L.S. Dang, Nature 443, 409 (2006).

25. T. Byrnes, N.Y. Kim, and Y. Yamamoto, Nat. Phys. 10, 803 (2014).

26. D. Bajoni, P. Senellart, E. Wertz, I. Sagnes, A. Miard, A Lemaître, and J. Bloch, Phys. Rev. Lett. 100, 047401 (2008).

27. J. Suffczyński, T. Kazimierczuk, M. Goryca, B. Piechal, A Trajnerowicz, K. Kowalik, P. Kossacki, A. Golnik, K.P. Korona, M. Nawrocki, J.A. Gaj, and G. Karczewski, Phys. Rev. B 74, 085319 (2006).

Publisher's Note Springer Nature remains neutral with regard to jurisdictional claims in published maps and institutional affiliations. 